\title{
Screening Cervical Spine CT in the Emergency Department, Phase 2: A Prospective Assessment of Use
}

\author{
B. Griffith, M. Kelly, P. Vallee, M. Slezak, J. Nagarwala, S. Krupp, C.P. Loeckner, L.R. Schultz, and R. Jain
}

\begin{abstract}
BACKGROUND AND PURPOSE: The National Emergency X-Radiography Utilization Study Low-Risk Criteria were established to identify patients with a low probability of cervical spine injury in whom imaging of the cervical spine was unnecessary. The purpose of this study was to ascertain the number of unnecessary cervical spine CT studies on the basis of proper application of established clinical guidelines and, secondarily, to determine indications for ordering studies in the absence of guideline criteria.
\end{abstract}

MATERIALS AND METHODS: All patients presenting to a level I trauma center for whom a screening cervical spine CT was ordered in the setting of blunt trauma were eligible for enrollment. For each study, the requesting clinician completed a survey regarding study indications. CT examinations were evaluated by a board-certified radiologist blinded to survey data to determine the presence or absence of cervical spine injury.

RESULTS: Of 507 CT examinations, 5 (1\%) were positive and 497 (98.0\%) were negative for acute cervical spine injury. Five studies (1\%) were indeterminate for acute injury but demonstrated no abnormality on subsequent imaging and clinical follow-up. Of the 502 studies without cervical spine injury, 81 (16.1\%) were imaged despite meeting all 5 NEXUS criteria for nonimaging. Of these, the most common study indication was dangerous mechanism of injury (48.1\%) followed by subjective neck pain (40.7\%).

CONCLUSIONS: Strict application of NEXUS criteria could potentially reduce the number of screening cervical spine CT scans in the setting of blunt trauma; this change would avoid a considerable amount of unnecessary radiation and cost.

ABBREVIATIONS: $C C R=$ Canadian Cervical Spine Rule; NEXUS = National Emergency X-Radiography Utilization Study; NLC = NEXUS Low-Risk Criteria; CSI = cervical spine injury

A nnually in the United States, $>1$ million patients will require assessment for potential cervical spine injury. ${ }^{1,2}$ Prior studies estimate that $2 \%-10 \%$ of these patients will have a CSI. ${ }^{3-5}$

There is constant and intensifying pressure on clinicians to assess and treat patients rapidly. Emergency departments face added strain due to the current health care climate: increased number of emergency department visits, fewer emergency departments, and high medical-legal exposure. ${ }^{6}$ These facts, coupled with increased accessibility and improving quality of medical imaging, may help explain the increased use of medical imaging in emergency departments. ${ }^{7}$

Use of clinical guidelines is one means of potentially curbing

Received May 7, 2012; accepted after revision July 4.

From the Departments of Radiology (B.G., M.K., R.J.), Emergency Medicine (P.V., M.S., J.N., S.K., C.P.L.), Public Health Sciences (L.R.S.), and Neurosurgery (R.J.), Henry Ford Health System, Detroit, Michigan.

Please address correspondence to Brent Griffith, MD, Department of Radiology, K3, Henry Ford Hospital, 2799 West Grand Blvd, Detroit, MI 48202; e-mail: brentg@ rad.hfh.edu

http://dx.doi.org/10.3174/ajnr.A3306 increased use of medical imaging. In 2000, the National Emergency X-Radiography Utilization Study Low-Risk Criteria were established to identify patients with a low probability of cervical spine injury in whom imaging of the cervical spine was unnecessary. ${ }^{8}$ The NEXUS criteria include the following: no tenderness at the posterior midline of the cervical spine, no focal neurologic deficit, normal level of alertness, no evidence of intoxication, and no clinically apparent painful injury that might distract a patient from the pain of a cervical spine injury. In 2001, a second decision rule, the Canadian Cervical Spine Rule was published. This rule uses 3 high-risk criteria (age 65 years or older, dangerous mechanism, paresthesias in the extremities), 5 low-risk criteria (simple rear-end motor vehicle crash, sitting position in emergency department, ambulatory at any time, delayed onset of neck pain, and absence of midline C-spine tenderness), and the ability of patients to actively rotate their necks, to determine the need for cervical spine radiography. ${ }^{9}$ Both the NEXUS and CCR criteria are included in the American College of Radiology appropriateness guidelines as a means of screening patients before imaging the cervical spine. $^{10}$ 
While these evidence-based decision rules are widely recognized, the degree of provider compliance has never been assessed. As a result, their impact on imaging is not well-defined. A retrospective study performed at our institution found that $23.9 \%$ of patients undergoing CT of the cervical spine following blunt trauma satisfied the 5 NEXUS criteria and should have avoided cervical spine imaging. ${ }^{11}$ However, given the inherent limitations of a retrospective review, including possible errors or omissions in the medical records, a collaborative prospective study between the departments of radiology and emergency medicine was undertaken. The purpose of this study was to prospectively determine the number of potentially avoidable cervical spine CT studies on the basis of proper application of established clinical guidelines. A secondary goal was to ascertain the indications used for ordering studies in the absence of guideline criteria.

\section{MATERIALS AND METHODS}

This Health Insurance Portability and Accountability Act-compliant prospective study was approved by our institutional review board. All patients presenting to the emergency department of a level I trauma center between March and November 2011, following blunt trauma, who underwent screening CT of the cervical spine as part of their evaluation were eligible for the study. According to the Trauma Practice Guidelines of our institution, CT is the recommended technique to assess cervical spine injury when imaging is clinically indicated. For each eligible patient, clinicians were instructed to complete a survey (Fig 1) documenting the following: mechanism of injury, indication for ordering the study, and clinical suspicion for cervical spine injury. Among the survey indications were the 5 NEXUS criteria, as well as an "abbreviated" set of CCR criteria, including age 65 years or older, dangerous mechanism, paresthesias in the extremities, and inability of the patient to actively rotate his or her neck. Due to the nature of the survey, we thought that, with the exception of midline C-spine tenderness, the 5 low-risk criteria (simple rear-end motor vehicle crash, sitting position in the emergency department, ambulatory at any time, delayed onset of neck pain, absence of midline Cspine tenderness) could not be accurately documented. In addition to the NEXUS and CCR criteria, clinicians could select from a number of other potential indications or could document their own indications. Triage acuity levels, which categorize patients according to their need for emergent medical intervention, were obtained for each enrolled patient.

CT examinations were evaluated by a board-certified radiologist blinded to the survey data to determine the presence or absence of cervical spine injury. As with our retrospective evalua-

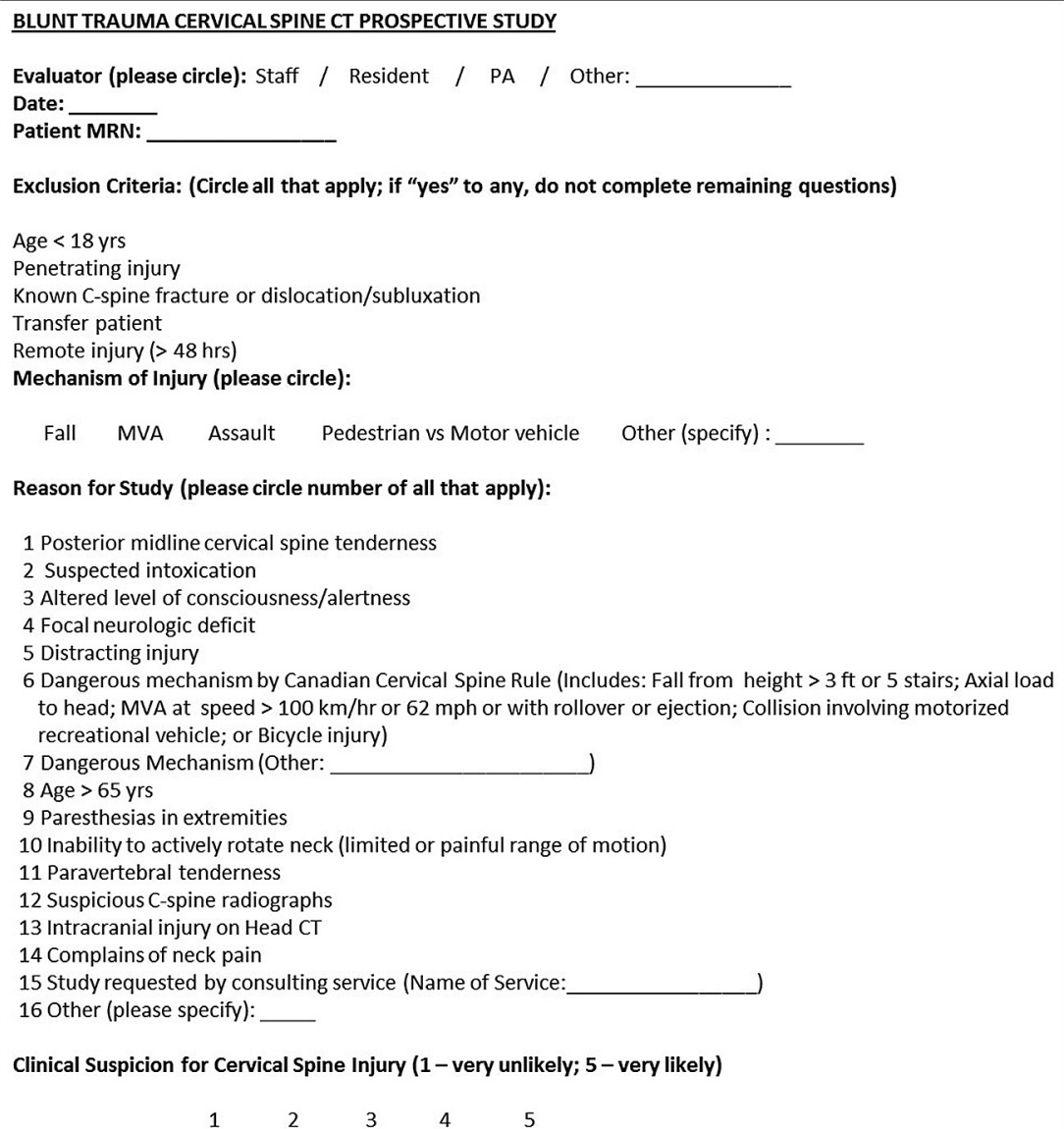

tion, a study with positive findings was one in which the radiologist's dictation indicated any fracture, dislocation, or subluxation based on CT findings. A study with negative findings had none of these indications. A study with indeterminate findings was one in which the radiologist suggested that a finding may be related to trauma or another cause. In these cases, further imaging and medical records were reviewed to confirm the finding.

The medical records of all patients undergoing cervical spine CT in the emergency department during the study period were analyzed by the study authors. A total of 1543 eligible patients were identified. Of these, 152 were excluded on the basis of the following exclusion criteria: younger than 18 years of age, penetrating trauma, transfer patient, remote injury of $>48$ hours, or known cervical spine fracture/dislocation/subluxation. Of the remaining 1391 eligible patients, 507 (36.5\%) were enrolled in the study. Surveys were not completed for the remaining 884 (63.5\%) patients (Fig 2 ). A subset of the nonenrolled patients was retrospectively reviewed to assess for selection bias.

\section{Statistical Methods}

When assessing the differences between patient subgroups for fracture rates, use of the NEXUS criteria, and overuse rates, we performed $\chi^{2}$ and Fisher exact tests. These tests were also used to compare patients enrolled in this study with a select group of patients not enrolled, to assess whether there was any selection bias. A 2 -sample $t$ test was used to compare age for these 2 groups 


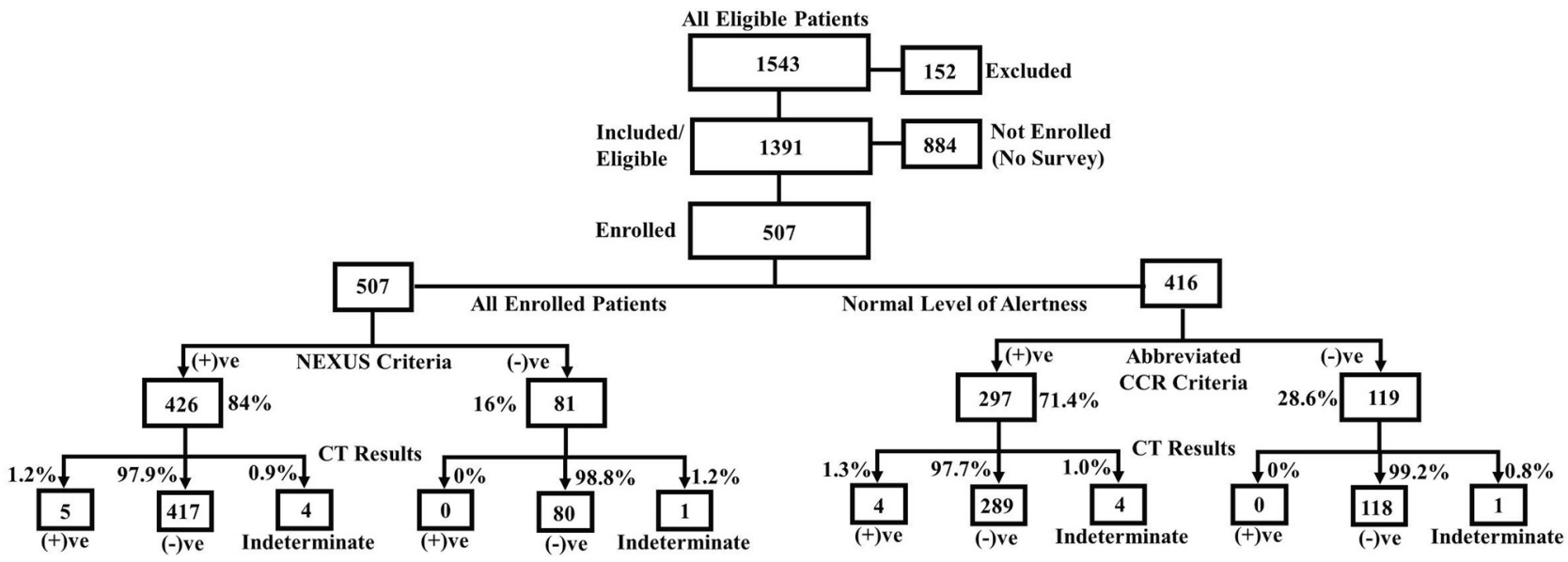

FIG 2. Flow diagram illustrates breakdown of study subjects according to National Emergency X-Radiography Utilization Study ${ }^{6}$ low-risk criteria, abbreviated Canadian Cervical Spine Rule criteria, and CT results.

Fracture Results: CT findings of subjects

\begin{tabular}{|c|c|c|c|c|}
\hline Studies & $\begin{array}{c}\text { Total } \\
\text { No. }\end{array}$ & $\begin{array}{l}\text { Negative Findings for } \\
\text { Acute Cervical } \\
\text { Spine Injury (No.) }\end{array}$ & $\begin{array}{l}\text { Positive Findings for } \\
\text { Acute Cervical } \\
\text { Spine Injury (No.) } \\
\end{array}$ & $\begin{array}{c}\text { Indeterminate Findings for } \\
\text { Cervical Spine Injury but } \\
\text { Negative Follow-up } \\
\text { Findings (No.) }\end{array}$ \\
\hline All Studies & 507 & $497(98.0 \%)$ & $5(1.0 \%)$ & $5(1.0 \%)$ \\
\hline Imaging appropriate by NEXUS criteria & 426 & $417(97.9 \%)$ & $5(1.2 \%$ & $4(1.0 \%)$ \\
\hline Imaging inappropriate by NEXUS criteria & 81 & $80(98.8 \%)$ & $0(0 \%)$ & $1(1.2 \%)$ \\
\hline Imaging appropriate by abbreviated CCR criteria & 297 & $289(97.7 \%)$ & $4(1.3 \%)$ & $4(1.0 \%)$ \\
\hline Imaging inappropriate by abbreviated CCR criteria & 119 & $118(99.2 \%)$ & $0(0 \%)$ & $1(0.8 \%)$ \\
\hline Imaging appropriate by CCR and/or NEXUS criteria & 469 & $459(97.9 \%)$ & $5(1.1 \%)$ & $5(1.1 \%)$ \\
\hline Imaging inappropriate by both NEXUS and CCR criteria & 38 & $38(100 \%)$ & $0(0 \%)$ & $0(0 \%)$ \\
\hline
\end{tabular}

of patients. The testing level was set at .05. All statistical analyses were done by using SAS, Version 9.2 (SAS Institute, Cary, North Carolina).

\section{RESULTS}

Of the 507 patients enrolled, 309 (60.9\%) were men and 198 (39.1\%) were women. The mean age of all patients was 44 years (age range, 18-100 years). Patients were triaged as follows: 218 (43\%) level one, 273 (53.8\%) level two, 15 (3\%) level 3, and 1 $(0.2 \%)$ with no recorded triage level. The most common mechanism of injury was motor vehicle crash (203/40\%), followed by a fall (150/29.6\%), assault (99/19.5\%), pedestrian versus motor vehicle (23/4.5\%), and other/not recorded (32/6.4\%).

Resident physicians completed the largest number of surveys (301/59.4\%), followed by senior staff physicians (115/22.7\%), and physician assistants (45/8.9\%). Forty-six (9.1\%) had no documentation of the completing personnel.

Of the 507 cervical spine CT examinations performed on enrolled patients, $5(1.0 \%)$ were positive for an acute cervical spine injury, and 497 (98.0\%) were negative. The remaining 5 (1.0\%) studies had indeterminate findings but failed to demonstrate an acute injury on subsequent imaging and clinical follow-up (Table).

Of the 502 examinations with no acute injury, 81 (16.1\%) met all 5 NEXUS criteria, and patients should not have been imaged. Four hundred twelve of these 502 examinations had no documented altered level of consciousness, thus making the patients eligible for screening with the CCR criteria. Of these 412, 119
(28.9\%) patients had none of the abbreviated CCR criteria and should not have been imaged. Overall, 38 (7.6\%) of the 502 patients without acute injury required no imaging when both the NEXUS and abbreviated CCR criteria were appropriately applied (Table).

Of the studies performed on patients who met all 5 NEXUS criteria (81 studies), 80 (98.8\%) were negative for acute cervical spine injury, none $(0 \%)$ were positive, and $1(1.2 \%)$ was indeterminate but failed to demonstrate acute injury either clinically or on follow-up imaging (Fig 2). Of those patients with no abbreviated CCR criteria (119 studies), 118 (99.2\%) were negative for acute cervical spine injury, none ( $0 \%)$ were positive, and $1(0.8 \%)$ was indeterminate but also failed to demonstrate acute injury either clinically or on follow-up imaging.

For patients defined as low risk by NEXUS and the abbreviated CCR criteria (38 patients), the most commonly cited indication for obtaining imaging was "complains of neck pain" (20/52.6\%), followed by "dangerous mechanism-non-CCR" (10/26.3\%), "consulting service requested" (5/13.2\%), "paravertebral tenderness" $(2 / 5.3 \%)$, "intracranial head injury on CT" $(1 / 2.6 \%)$, and "other" (2/5.3\%).

For appropriately ordered studies based on NEXUS criteria (426 patients), the most commonly documented criterion was posterior midline cervical spine tenderness (237/55.6\%), followed by suspected intoxication (174/40.8\%), altered level of consciousness/alertness (91/21.4\%), distracting injury (87/20.4\%), and focal neurologic deficit (14/3.3\%). The most commonly doc- 
umented indication for imaging, according to the CCR criteria (297 patients), was posterior midline tenderness (222/74.7\%), followed by dangerous mechanism of injury (87/29.3\%), older than 65 years $(48 / 16.2 \%)$, paresthesias in the extremities (20/ $6.7 \%)$, and inability to actively rotate the neck/limited or painful range of motion $(19 / 6.4 \%)$.

When investigators reviewed the survey tool, it was noted that staff physicians did not follow NEXUS guidelines in $9.6 \%$ of cases, while residents and physician assistants did not follow guidelines in $16.9 \%$ and $20 \%$ of cases, respectively.

A subset analysis was performed on 100 consecutive eligible but nonenrolled patients to look for selection bias. This analysis found no statistically significant difference between patients included in the study and those for whom surveys were not completed on the basis of age $(P=.652)$, sex $(P=.910)$, triage level $(P=.362)$, mechanism of injury $(P=.477)$, and fracture rates $(P=.455)$.

\section{DISCUSSION}

The goals of the NEXUS Low-Risk Criteria and Canadian Cervical Spine Rule are to identify trauma patients with low probabilities of cervical spine injury, thereby sparing them unnecessary cervical spine imaging. ${ }^{2,8,9,12}$ To meet the NEXUS criteria, a patient must have the following: no tenderness at the posterior midline of the cervical spine, no focal neurologic deficit, normal level of alertness, no evidence of intoxication, and no clinically apparent painful injury that might distract him or her from the pain of a cervical spine injury. ${ }^{8}$ The Canadian Cervical Spine Rule uses 3 high-risk criteria (age 65 years or older, dangerous mechanism, paresthesias in extremities), 5 low-risk criteria (simple rear-end motor vehicle crash, sitting position in the emergency department, ambulatory at any time, delayed onset of neck pain, absence of midline Cspine tenderness), and the ability of patients to actively rotate their necks, to determine the need for cervical spine radiography. ${ }^{9}$ Both the NEXUS criteria and CCR criteria were validated in large studies, with reported sensitivities and specificities of $99 \%$ and $12.9 \%$, respectively, in the NEXUS study and $100 \%$ and $42.5 \%$ in the CCR study. Subsequent studies have questioned the sensitivity of these criteria, particularly the NEXUS criteria. ${ }^{2,11,12}$ A study by Duane et $\mathrm{al}^{13}$ in 2007 suggested that a clinical examination cannot reliably diagnose or exclude cervical spine fracture in patients with blunt trauma. Despite this suggestion, both NEXUS and CCR are still widely used, and the American College of Radiology accepts both criteria in their appropriateness guidelines as a means of screening patients before imaging the cervical spine. ${ }^{9}$

Despite widespread knowledge of these guidelines, emergency physicians still have a low threshold for ordering cervical spine imaging. ${ }^{8}$ While part of this practice may relate to disastrous consequences from missed cervical spine injuries, it also likely stems from both increasing demands on physicians to make quick and accurate diagnoses, as well as the availability, speed, and improved diagnostic accuracy of imaging modalities.

The retrospective study performed at our institution found that strict application of NEXUS criteria before cervical spine imaging would have resulted in a $23.9 \%$ reduction in the number of cervical spine CT scans with negative findings in patients presenting with blunt trauma. ${ }^{11}$ However, because that study was based on retrospective chart review, the data were limited to information recorded by medical personnel at the time of the patient's presentation. This limitation resulted in uncertainty about the accuracy of the calculated rate of overuse. For this reason, a prospective study was undertaken to provide a more accurate estimate. The results of this study found that strict application of NEXUS criteria before cervical spine imaging would have resulted in a $16.1 \%$ reduction in the number of cervical spine CT studies with negative findings. This is a decrease from the $23.9 \%$ found in the retrospective study $(P<.001)$. Similarly, application of the abbreviated CCR criteria to appropriate cases would have resulted in a $28.9 \%$ reduction in the number of studies with negative findings. Application of both clinical criteria would have resulted in a reduction of only $7.6 \%$ because applying 2 separate screening criteria simultaneously greatly decreases the specificity of the criteria and leads to a substantial increase in the number of unnecessary studies. However, even if one assumed a 7.6\% reduction in imaging, this would still represent considerable savings in both radiation exposure and health care costs, considering the $>1$ million patients with blunt trauma presenting annually in the United States.

Gaining an understanding of the indications cited for cervical spine imaging that are not part of accepted guidelines may help identify potentially remediable "patterns" in overuse. In our study, the most commonly cited indication for imaging in the absence of both the NEXUS and CCR criteria (38 studies) was "complains of neck pain" (20/52.6\%), followed by "dangerous mechanism-non-CCR" (11/28.9\%), and "consulting service requested" (5/13.2\%). By identifying these trends, clinicians may be more confident in clearing the cervical spine when strict clinical guidelines are applied and can be more confident in not ordering medical imaging when other nonguideline clinical findings exist.

The study also found that while staff emergency department clinicians ordered studies in the absence of the NEXUS criteria in $9.6 \%$ of cases, residents and physicians assistants ordered studies without NEXUS criteria in $17.3 \%$ of cases $(P=.045)$. These differences may relate to better understanding of the clinical criteria and their application in the setting of blunt trauma and/or better clinical assessment skills in more experienced care providers.

The obvious downside to strict application of clinical criteria for ordering screening CT examinations is the potential for missed injuries. While 4 patients with cervical spine injury met NEXUS criteria for foregoing imaging in the retrospective study, ${ }^{11}$ proper application of both the NEXUS and abbreviated CCR criteria allowed detection of all patients with cervical spine injury in the current study. Moreover, while this study was not designed to test the sensitivity of either criteria, both the NEXUS and CCR had sensitivities of $100 \%$ for detecting cervical spine injury in the imaged population.

The greatest limitation of our study was low patient enrollment. Of the 1391 patients eligible for inclusion in the study, only $36.5 \%$ were enrolled. Surveys were not completed for the remaining $63.5 \%$ patients. This rate likely relates to the setting in which the study was performed, a level I trauma center, where clinicians frequently do not have time or do not remember to fill out a survey. Due to concern for selection bias, a subset analysis was performed to analyze characteristics of enrolled and nonenrolled 
patients. It demonstrated no statistically significant difference between the 2 groups.

A second limitation of the study is that the CCR criteria were not fully interrogated. Instead, a portion of the "low-risk" criteria (simple rear-end motor vehicle crash, sitting position in emergency department, ambulatory at any time, delayed onset of neck pain) were excluded because it was decided that these would be difficult to document on the survey form. However, even with the exclusion of these criteria, no acute cervical spine injuries were missed.

A third potential limitation is that the simple act of introducing a survey regarding the indication for the study may have led to a change in ordering practices. This may explain some of the differences noted in the reported rate of overuse in the retrospective study $(23.9 \%)^{11}$ and the current prospective study (16.1\%).

Finally, direct comparison of the results of this prospective study with the results of the prior retrospective study is difficult. The "overuse" rate determined from the retrospective study may be overestimated if screening clinical criteria were not accurately documented in the medical record.

The next phase of this study will involve an education program for clinicians in the emergency department regarding the appropriate use of accepted clinical guidelines in determining the need for screening cervical spine CT in the setting of blunt trauma. This will then be followed by another prospective study to determine whether the educational intervention results in changes in ordering practices and improvement in the use of the imaging services.

\section{CONCLUSIONS}

Within the limits of this study, our findings reveal that many patients undergo imaging despite meeting guideline criteria for nonimaging. Determining a definite cause for the overuse is difficult, but possibilities include the following: lack of knowledge regarding the clinical guidelines, lack of trust in the guidelines to accurately predict injury, and complex guidelines that are difficult to apply or interpret. Given the statistically significant discrepancy between ordering practices of staff physicians and residents/ physician assistants, the study also suggests that further education, especially of residents and midlevel providers, regarding clinical guidelines may improve adherence and decrease overuse.

This collaborative effort between the departments of radiology and emergency medicine demonstrates the potential for substan- tial improvement in use. With a nation focused on improving the use of health care resources, as well as decreasing radiation exposure, the burden is on both radiologists and clinicians to address appropriate use of all imaging modalities.

\section{REFERENCES}

1. Pitts SR, Niska RW, Xu J, et al. National Hospital Ambulatory Medical Care Survey: 2006 Emergency Department Summary — National Health Statistics Reports; No 7. Hyattsville, Maryland: National Center for Health Statistics; 2008

2. Dickinson G, Stiell IG, Schull M, et al. Retrospective application of the NEXUS low-risk criteria for cervical spine radiography in Canadian emergency departments. Ann Emerg Med 2004;43:507-14

3. Bailitz J, Starr F, Beecroft M, et al. CT should replace three-view radiographs as the initial screening test in patients at high, moderate, and low risk for blunt cervical spine injury: a prospective comparison. J Trauma 2009;66:1605-09

4. Greenbaum J, Walters N, Levy PD. An evidence based approach to radiographic assessment of cervical spine injuries in the emergency department. J Emerg Med 2009;36:64-71

5. Griffen MM, Frykberg ER, Kerwin AJ, et al. Radiographic clearance of blunt cervical spine injury: plain radiograph or computed tomography scan? J Trauma 2003;55:222-26, discussion 226-27

6. Committee on the Future of Emergency Care in the United States Health System. Hospital-Based Emergency Care: At the Breaking Point. Washington, DC: National Academies Press; 2007:19-23

7. Korley FK, Pham JC, Kirsch TD. Use of advanced radiology during visits to US emergency departments for injury-related conditions, 1998-2007. JAMA 2010;304:1465-71

8. Hoffman JR, Mower WR, Wolfson AB, et al. Validity of a set of clinical criteria to rule out injury to the cervical spine in patients with blunt trauma: National Emergency X-Radiography Utilization Study Group. N Engl J Med 2000;343:94-99

9. Stiell IG, Wells GA, Vandemheen KL, et al. The Canadian C-spine rule for radiography in alert and stable trauma patients. JAMA 2001;286:1841-48

10. Daffner RH, Hackney DB. ACR appropriateness criteria on suspected spine trauma. J Am Coll Radiol 2007;4:762-75

11. Griffith B, Bolton C, Goyal N, et al. Screening cervical spine CT in a level I trauma center: overutilization? AJR Am J Roentgenol 2011; 197:463-67

12. Stiell IG, Clement CM, McKnight RD, et al. The Canadian C-spine rule versus the NEXUS low risk criteria in patients with trauma. N Engl J Med 2003;349:2510-18

13. Duane TM, Dechert T, Wolfe LG, et al. Clinical examination and its reliability in identifying cervical spine fractures. J Trauma 2007;62: 1405-10 